\title{
Is Multibeam Water Column Data Really Worth the Disk Space?
}

\author{
Authors: GEE Lindsay ${ }^{1}$, DOUCET Maurice ${ }^{1}$, PARKER David ${ }^{2}$, WEBER Tom ${ }^{3}$, and BEAUDOIN
} Jonathan ${ }^{3}$

1. Quality Positioning Services, Inc., 104 Congress Street, Portsmouth NH, USA

2. United Kingdom Hydrographic Office (UKHO), Admiralty Way, Taunton, Somerset, TA12DN, UK

3. Center for Coastal and Ocean Mapping/Joint Hydrographic Center (CCOM-

JHC), University of New Hampshire, 24 Colovos Road, Durham NH, USA

Topic: C. Innovations in Processing Techniques

\section{INTRODUCTION}

The very latest multibeam sonars have the capability to record the sonar time series for each beam; mapping the water column along with the seafloor. This capability opens up new uses for multibeam sonars, including the direct mapping of fish and marine mammals, the mapping of plumes and vents, the location of mid-water targets, and a wide range of physical oceanographic processes. To exploit these data, an efficient means of reading, processing and analyzing the data is required. This presentation describes the ongoing development of a commercial tool (FMMidwater) to make these data available for hydrographic surveyors, scientists, and engineers interested in visualization and analysis of water column data, in a multi-mode 4D environment.

\section{DEVELOPMENT CHALLENGES}

A number of challenges needed to be addressed during the development of commercial software tools to allow efficient exploitation of the water column data.

Each sonar manufacturer has a specific data logging file format that is used to capture water column data, and the lack of a standard sonar format provided a challenge for development. Each logged file or files contains data from a specific component within the system such as navigation, attitude, backscatter, multibeam and water column data. A single water column ping may be fragmented across multiple packets; this can be further complicated when navigation and attitude information are logged in separate packets, or in some cases logged to a completely separate file. This results in fragmentation of an instantaneous snapshot of the water column. In order to compute where each sample within a single ping exists in time and space, we need to integrate the information from these different packets/files.

Another significant challenge are the high data rates that exist in some of the shallow water multibeam sonars types, that results in an order of magnitude additional water column data above that from standard bottom detection. Historically there has been an increase in data rates for multibeam sonars as ping rates and number of beams increased, and these data rates can be expected to continue to rise. In order for data analysis and visualization to be efficient and interactive, data loading must be fast with a minimum amount of disk seek time and read delays. Noting the above challenges, it was necessary to avoid the loading of data from very large fragmented files.

The initial development of FMMidwater was built on a research prototype (GeoZui4D) developed at the University of New Hampshire to investigate methods for interactively visualizing time varying geospatial data [Arsenault et al, 2004; Ware et al, 2001] [1][2]. Water column data required a number of new visualization metaphors and export options beyond those used for bathymetry data, in order to address the geospatial and temporal aspects of the analysis, and to extract fully refracted and adjusted detections to fuse with seabed detections from the multibeam processing and visualization. 
Finally, it was necessary to ensure that flexibility was incorporated in the tool to allow for both the simple addition of evolving data types as more multibeam sonars provided the water column data, and to facilitate the many emerging uses and users of the data.

\section{UNIFIED GENERIC WATER COLUMN FORMAT}

Addressing the primary challenges that exist in exploitation of water column data, an open generic water column format (GWC) was designed as a unified way of storing water column data in a compressed or sub-sampled, integrated manner to be used for efficient interactive visualization [3]. The GWC can be considered analogous to the GSF format [4] that is used for storing processed and raw bathymetry. The open format GWC also assists in meeting the challenge of the evolving multibeam data formats by providing a path for third party developers, such as sonar or survey companies, and researchers, to easily and quickly add format support to FMMidwater.

In the FMMidwater tool the water column packets, time-based navigation and attitude are reintegrated during source conversion, such that all the relevant data of any particular ping is available. The tool also allows the integration of separate navigation and attitude files that may have been processed separately such as with ROV and AUV operations. Files are indexed for fast non-linear extraction and every packet type is extracted for relevant information into the GWC format. Currently, the source conversion system supports a wide variety of hydrographic and fisheries sonars such as the Kongsberg EM series sonars, ME70, EK60, EK500, R2Sonic 2024 and Reson 7125.

\section{FEATURE EXTRACTION}

The raw data rates of water column data collected from multibeam sonars are very high, however the information content is generally much smaller. An important aspect of any tool used to examine these data is the ability to rapidly review the data and extract features of interest. FMMidwater provides multiple ways to view and threshold the data for display and analysis; swath view, beam view, and stacked view, and a combination of these.

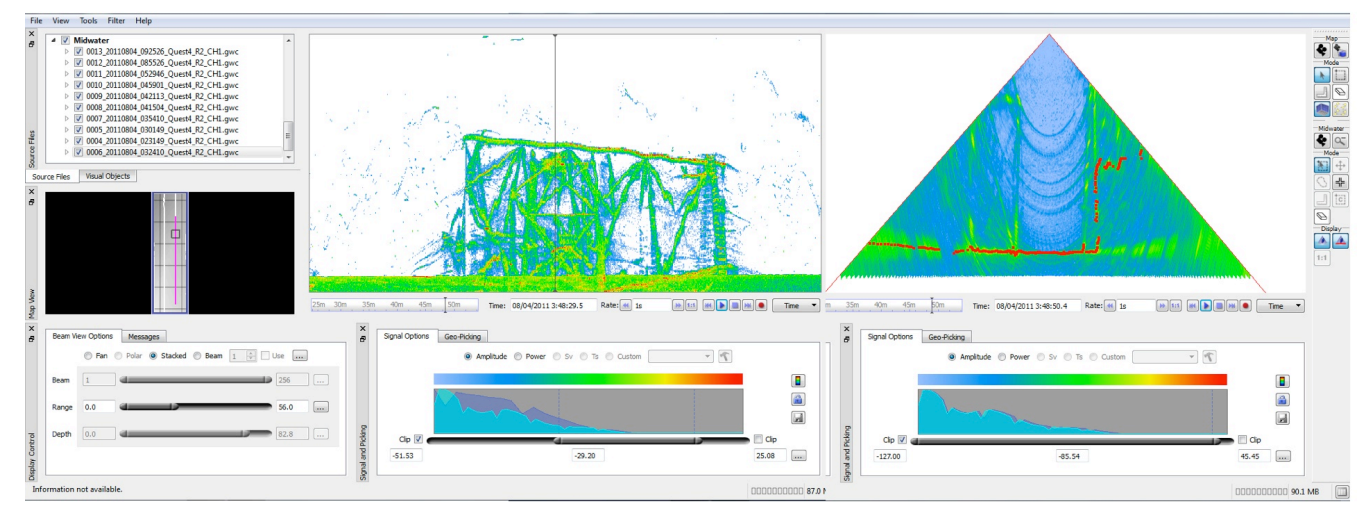

Figure 1. - FMMidwater Stacked and Fan Views - with red points from bottom online detection

The various views allow easy and rapid identification of features for selection, threshold filtering and extraction. The various views also allow direct comparisons of the water column data with the online bottom detection, and easy identification of detection failures or errors in the hydrographic multibeam sonars. Filters can be applied to specifically focus on certain beams and to limit the range of data used. In addition, the threshold adjustment can be applied to the sonar signal itself. Each GWC file also generates a histogram of the data during the load process. A control is provided such that the user can eliminate low-level noise and either clamp or clip the data at either end of the threshold limits. The histogram can display raw amplitude, power, volume scattering or target strength depending on the capabilities of 
the sonar. Interactive digitizing can be used during the final selection to constrain the area of the data to be extracted.

\section{VISUALIZATION AND ANALYSIS}

After focusing on the features in the water column in FMMidwater, the contacts can be exported for visualization and further analysis in the Fledermaus $4 \mathrm{D}$ environment. Four primary visualization metaphors were developed to provide the most effective means of reviewing water column features:

- Beam Fan Time Series object - a view of the swath that is temporally aware and rendered dynamically in the 4D environment from the GWC file

- Beam line object - a time-aware along-track curtain, or image fence, from an individual beam

- Point cloud object - a time aware object of extracted points with transformed location of the sample in the beam with attributes for line/beam and signal level.

- Volume object - a 3D grid of voxels at a specified cell size. In Fledermaus the voxel brick is used to generate ISO surfaces at user selected values.

The water column data can be used in hydrographic surveying for ensuring that hard targets, such as wrecks are fully detected, and to confirm the least depth in the water column versus the bottom detection. This is achieved in both FMMidwater and also in the Fledermaus PFM data object for integrated multibeam validation and analysis. In the following figures, data is from a Kongsberg EM2040 mounted on an ROV during a survey in the Gulf of Mexico.

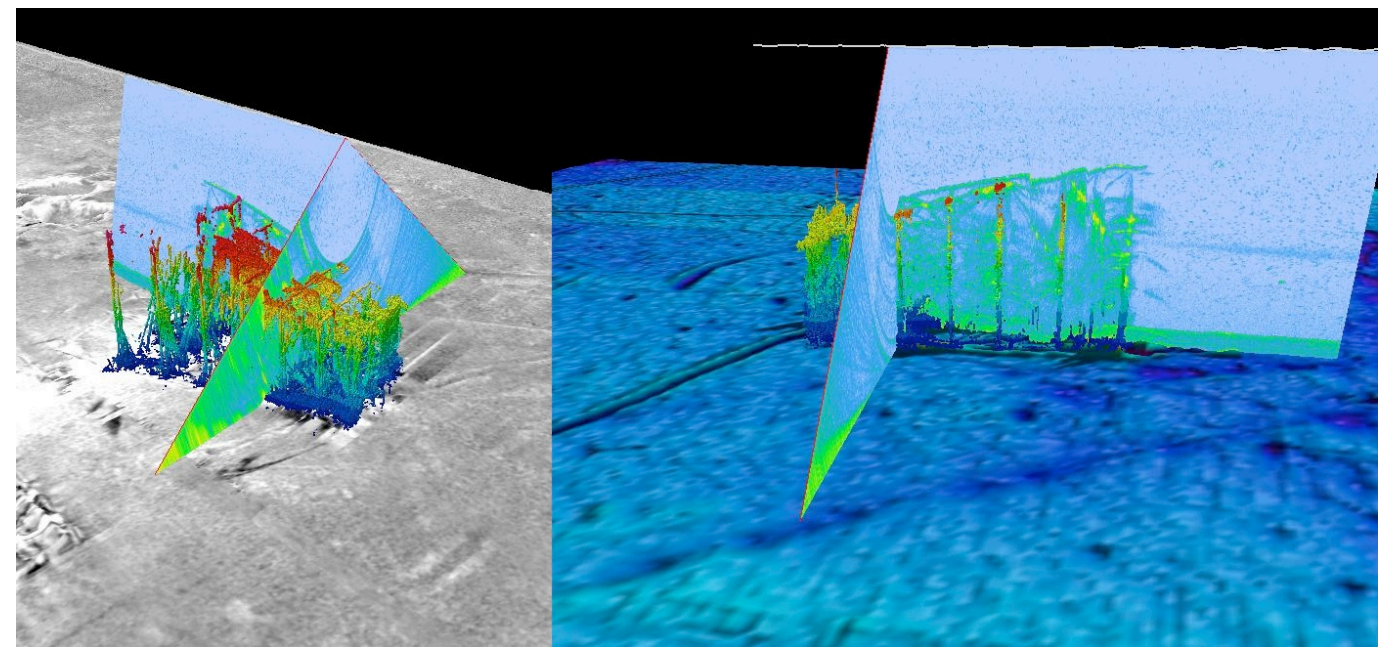

Figure 2. - Fledermaus fan and beam curtain objects with bottom detected points and seabed grid. Fan and beam show additional contacts in the water column data.

\section{WATER COLUMN TOOL AND PLUG INS}

The water column capability of modern multibeam sonars has widened the applications for water column data and has the potential to extend the capabilities of the multibeam sonars in various fields such as hydrographic survey, resource exploration, and marine habitat mapping. This presents a significant challenge in developing software applications that can address all of these potential user requirements. One option was to restrict the development of the mid-water tool to a particular area such as hydrographic surveying. Instead, FMMidwater has been structured to allow it to become a platform for processing water column data, with the flexibility to extend the baseline capabilities to meet specific user requirements by adding plugins. This model of extending software applications with new runtime capabilities is well used by the more traditional software market, however it is new in the market covered by maritime processing software. Plugins are a means of adding specific capabilities to a larger baseline application without the need of direct integration that would require a new build or release of that application. Just as important is that third party 
companies, universities, research institutions or singular individuals can offer the plugins they develop directly to other users. The plugins intellectual property can be maintained by the authoring organization and either given away or sold and supported just like traditional software. This approach addresses the significant problem of bringing prototype developments from research to a broader community of users, without the researcher being required to develop and support an entire software application.

QPS began this approach with the Fledermaus Geocoder Toolbox (FMGT) for much the same reasons outlined for FMMidwater [5]. The original Geocoder research software was retooled, or refactored, into a robust, stable and modular software framework. A key in allowing external access was the retooling of the Geocoder processing pipelines into software modules; these modules honor the algorithms implemented in the original Geocoder framework but with clear boundaries being set between the various data flows and processing stages such that researchers can investigate and potentially improve upon a single module without the overhead of maintaining the overall software framework. Further architecture modifications allowed for swappable plugin functionality for the core routines such that researchers could focus on a specific stage of processing without having to rebuild and support the entire research application.

Plugins within the FMMidwater platform are broken down into a number of major types:

- Import - transform external data into formats for GWC files.

- SD Export - transform GWC data into other visualization objects.

- Data Export - transform GWC data into other custom formats, with or without additional processing. The first implementation of data refraction used this type of plugin. Another was jointly developed with the Royal Netherlands Institute for Sea Research (NIOZ) and CCOM UNH; this plugin allows split-beam processing when exporting targets from the Simrad single beam series of fisheries sonars.

- Signal Processing - to transform the raw GWC time series data into some other domain such as volume scattering or target strength.

- Tools - any type of custom operation that can be added such as a datagram or navigation plotting viewer.

- Context Menu- perform specific operations on a selected file from the file tree view.

When a plugin is installed on the system, FMMidwater will interrogate it during startup for its specific type. Depending on the type, the plugin will be attached to a particular part of the FMMidwater user interface.

\section{HYDROGRAPHIC APPLICATION - WRECK INVESTIGATIONS}

For many years the UK Civil Hydrography Programme (CHP) specification has required survey contractors to undertake investigations of all wrecks in a given survey area using both side scan sonar and extra multibeam sonar survey lines. For critical wrecks, wire sweeping has also been required. For a number of years there has been concern as to whether multibeam sonars are able to safely determine least depth when fine features (e.g. masts) may protrude from a wreck. The capability of applications like FMMidwater now allows the processing of water column data and comparison against the bathymetry from conventional online bottom detection. This capability means that multiple features within each receive beam may be digitized, rather than one single solution, and also that very fine features within the water column - finer than would ordinarily be seen in conventional bathymetry - should be detectable, especially in post-processing.

A field trial was undertaken with one of the CHP Contractors (Fugro OSAE) to compare the results obtained from these different methods; multibeam bathymetry, sidescan, wire sweeping and water column processing. It was hoped that the trial would confirm that logging and processing water column data alongside conventional bottom detection data could meet all wreck investigation requirements. The following three multibeam sonars were compared; Kongsberg EM3002, Kongsberg EM 710, and Reson SeaBat 7125. 
Sixteen targets were investigated with each acoustic method, and five of these targets were also wire swept. On eight of the targets, the minimum least depth by acoustic methods was indicated by water column data; although only two of these were of real significance, being up to $2.6 \mathrm{~m}$ shoaler than the online bottom detection, as seen in Table 1 . One target was a $3 \mathrm{~m}$ upright pole placed on the seabed. None of the multibeam sonar detected this feature in the conventional online bottom detection, but it was clearly visible in the water column data.

\begin{tabular}{|c|c|c|c|c|c|c|c|}
\hline No. & Wreck-Name & $\begin{array}{c}\text { General depth } \\
{[\mathrm{m}]}\end{array}$ & $\begin{array}{c}\text { MBES } \\
\text { least depth } \\
{[\mathrm{m}]}\end{array}$ & $\begin{array}{c}\text { WCD } \\
\text { least depth } \\
{[\mathrm{m}]}\end{array}$ & $\begin{array}{c}\text { SSS } \\
\text { least depth } \\
{[\mathrm{m}]}\end{array}$ & $\begin{array}{c}\text { Wire sweep } \\
\text { cleared depth } \\
{[\mathrm{m}]}\end{array}$ & $\begin{array}{c}\text { Difference from } \\
\text { Next Shoalest } \\
{[\mathrm{m}]}\end{array}$ \\
\hline 1 & Hellopes Boiler & 34.00 & 28.6 & 28.6 & 28.6 & - & 0.0 \\
\hline 2 & T-FG-009 & 37.30 & 36.0 & 35.9 & 36.0 & - & 0.1 \\
\hline 3 & T-FG-014 & 34.20 & 30.3 & 30.1 & 30.3 & - & 0.2 \\
\hline 4 & T-FG-016 & 37.00 & 29.6 & 29.6 & 29.6 & - & 0.0 \\
\hline 5 & T-HB-018 & 43.00 & 38.2 & 38.0 & 38.2 & - & 0.2 \\
\hline 6 & T-VH-003 & 41.00 & 35.6 & 35.6 & 35.3 & - & 0.3 \\
\hline 7 & W-16309 & 40.00 & 31.6 & 31.8 & 31.8 & - & 0.2 \\
\hline 10 & W-16400 & 28.90 & 20.3 & 20.4 & 20.8 & - & 0.1 \\
\hline 11 & W-16445 & 40.00 & 34.3 & 32.6 & 34.3 & - & 1.7 \\
\hline 12 & W-16515 & 39.00 & 32.3 & 32.2 & 32.3 & - & 0.2 \\
\hline 14 & W-17789 & 33.00 & 27.4 & 24.8 & 27.3 & - & 2.6 \\
\hline 16 & W-16439 & 35.00 & 30.2 & 30.2 & 30.2 & & - \\
\hline 17 & Hellopes N & 33.60 & 27.1 & 27.8 & 26.6 & 27.3 & 0.0 \\
\hline 18 & Hellopes S & 34.00 & 30.0 & 30.0 & 30.0 & 29.5 & 0.5 \\
\hline 19 & W-73522 & 42.40 & 35.4 & 35.7 & 35.9 & 34.9 & 0.5 \\
\hline 20 & W-73523 & 39.00 & 35.9 & 35.8 & 36.0 & 35.4 & 0.5 \\
\hline 21 & EM710-Frame & 18.80 & $18.8 *$ & 15.2 & - & 15.6 & 0.1 \\
\hline
\end{tabular}

Table 1. - Results from field trial (UK Hydrographic Office and Maritime and Coastguard Agency)

The conclusion from this initial trial was that in general, all systems gave similar results in most cases when the uncertainty of each method was considered. However, for some features the water column data did appear to detect features that were undetected in the other acoustic datasets. It was also found that even with excellent equipment, it was very hard to get good repeatability from wire sweeping. Following these conclusions, the CHP Specification now requires contractors to gather multibeam sonar water column data during all wreck investigation lines, then review this data against the online bottom detection. If shoaler wreck features are found in the water column data, then a new depth is to be selected by the hydrographer from the water column and incorporated into the dataset.

\section{CONCLUSION}

Mid-water capable sonars have been available for several years, but until recently there have been very few commercial tools to analyze and extract information from these data outside of the initial 2D view during acquisition. In 2009, FMMidwater, a commercial off-the-shelf module for post-processing and visualizing water column data was released. The software, developed in conjunction with researchers at the Center for Coastal \& Ocean Mapping, University of New Hampshire, provides a tool to make use of this new data type, allowing for the extraction of fully refracted and adjusted detections to fuse with seabed detections from the multibeam sonar, for further processing and visualization.

This capability has widened the applications for water column data and has the potential to extend the capabilities of the multibeam sonars in various fields such as hydrographic survey, resource exploration, and marine habitat mapping. The challenge of addressing all the new data from multibeam sonars with water column capability and the many emerging users is being addressed by developing a software platform that supports plugins. The plugin approach is a new paradigm for maritime processing software and provides significant advantage for third party users, developers and researchers by providing a flexible solution to add additional functionality to a robust software platform. 
The trial by the UK Civil Hydrography Programme (CHP) using water column data and the modification of their specifications provides a good example of the emerging use of these data and developing applications. The use of an off-the-shelf module such as FMMidwater now allows extraction of fully corrected and refracted multiple returns from each beam, which can be used for the detection and definition of fine targets such as masts on wrecks.

\section{ACKNOWLEDGMENT}

The authors gratefully acknowledge the support of the initial development by a grant from the New Hampshire Innovation Research Center. Thanks also to Kongsberg Maritime for providing the EM2040 data of the oil rig water column sample survey.

\section{REFERENCES}

[1] Arsenault, R., Ware, C. , Plumlee, M., Martin, S. C., Whitcombe, L. L., Wiley, D. N., Gross, T., Bilgili, A. , 2004, "A system for visualizing time-varying oceanographic 3D data", IEEE Oceans, Halifax, Nova Scotia, Canada, 1 - 5 October, 5 pages. Conference Proceeding. [2] Ware, C. , Plumlee, M. , Arsenault, R. , Mayer, L. A., Smith, S. , House, D. H., 2001, "GeoZui3D: Data Fusion for Interpreting Oceanographic Data", IEEE Oceans, Honolulu, HI, USA, 5 - 8 November, pp. 2498 - 2502. Conference Proceeding.

[3] Doucet, M., Arsenault, R., Ware, C., Weber, T., Malik, M., Mayer, L., Gee, L., 2009 "Advanced Mid-Water Tools for 4D Marine Data Fusion and Analysis", IEEE Oceans, Biloxi, MS, USA, 26-29 October, 9 pages. Conference Proceeding.

[4] SAIC, Generic Sensor Format, version 3.0, April 2009.

[5] Doucet, M., Paton, M., Calder, B., 2011 "From Research to Reality; Geocoder's Past, Present, and Future", USHydro2011, Tampa, FL, USA, 25-28 April. Conference Proceeding. 